\title{
PRODUCCIÓN DE LECHE Y PORCENTAJE DE GRASA EN EL DÍA DE CONTROL DE BÚFALAS DE RÍO EN LA PROVINCIA GRANMA
}

\author{
MILK YIELD AND FAT PERCENTAGE AT THE CONTROL DAY IN RIVER \\ BUFFALO COWS IN THE GRANMA PROVINCE
}

\author{
Méndez, M. ${ }^{1 *}$ y Fraga, L.M. ${ }^{1}$ \\ ${ }^{1}$ Instituto de Ciencia Animal. San José de las Lajas. La Habana. Cuba. *mmendez@ica.co.cu
}

\author{
Palabras clave adicionales \\ Modelo. Curva. Repetibilidad.
}

\section{RESUMEN}

Se estudió el comportamiento de la producción de leche (PDCL) y porcentaje de grasa (PDCG) en el día de control de rebaños bubalinos de la provincia Granma con 2140 controles mensuales de producción diaria de leche y 1620 de grasa de 500 lactancias, correspondientes a 150 búfalas de la raza Buffalypso, cuyos partos se efectuaron entre los años 1997 a 2005. Se utilizó el paquete estadístico SAS con empleo de un modelo mixto de efectos fijos, con los efectos grupo de contemporáneas, fecha de pesaje, y la interacción entre rebaño y fecha de pesaje, como fijos y como aleatorios, rebaño y arete de cada animal anidado dentro de cada rebaño. Se calcularon los valores de repetibilidad para leche y grasa. Se concluye que la producción de leche en el día de control de las búfalas fue baja y el porcentaje de grasa se mantuvo dentro del rango para esta especie. Las repetibilidades determinadas de forma general se encuentran dentro del rango de medio a alta. La modelación con los valores PDCL y PDCG ajustados por el modelo lineal tuvieron coeficientes de ajustes altos con el empleo del modelo cuadrático logarítmico por lo que se recomienda que la selección de búfalas para producción lechera se realice a partir de los registros repetidos del día de control por los valores más altos de repetibilidades obtenidos y estudiar los factores no genéticos que pudieran estar afectando la producción lechera.

\section{SUMMARY}

The performance of milk yield (PDCL) and fat percentage (PDCG) was studied in the control day

\section{AdDitional KeYWORDS}

Model. Curve. Repetibility.

in buffalo herds of the Granma province using 2140 monthly records of daily milk yield and 1620 of fat records from 500 lactations. This corresponded to 150 buffalo cows of the Buffalypso breed whose calvings occurred between 1997 and 2005. The SAS statistical software was used with a mixed model of fixed effects for the effects group of contemporaries, weighting date and the interaction between herd and weighting date as fixed, and herd and earring of each animal within each herd, as random. The repeatability values for milk and fat were calculated. It was concluded that the milk yield in the control day was low and the fat percentage was kept within the range for this species. The repeatabilities were, in general, within the range from middle to high. The modeling with the values TDMP and TDMF fitted by the lineal model had high coefficients of adjustment using the logarithmic quadratic model. The selection of the buffalo cows for milk yield could be done from the repeated records of the control day through the highest values of repeatabilities obtained, studying also the non-genetic factors affecting milk yield.

\section{INTRODUCCIÓN}

Desde 1983 a 2008, la masa bubalina en Cuba creció de 120 animales a 63 050, representando las hembras un $17,6 \%$, demostrando su capacidad reproductiva y alto nivel de supervivencia. Esto unido a la calidad nutritiva y valor industrial de sus pro- 


\section{MÉNDEZYFRAGA}

ducciones los convierte en una excelente opción para la producción de alimentos para el consumo humano.

En 1997 se comenzaron a establecer lecherías en la provincia de Granma con la entrada de 30 animales en una lechería. A partir de entonces esta especie se desarrolló de manera vertiginosa, pero aún con técnicas muy rudimentarias, sobre todo en esta zona oriental del país, que junto con el nuevo impulso de reestructuración de los CAI (complejo agroindustrial) azucareros para la diversificación agropecuaria y sumado a los esfuerzos de las empresas especializadas, la provincia ha alcanzando la cifra de 1610 búfalos en 14 unidades pertenecientes tanto al MINAGRI (Ministerio de la Agricultura) como al MINAZ (Ministerio de la Industria Azucarera).

La leche de búfala, por su alto contenido de grasa, tiene un alto valor energético, la proteína contiene más caseína y ligeramente más albúmina y globulinas que la de vaca (García y Planas, 2003). De acuerdo con Hernández y Espinosa (2005), en Cuba se obtienen $830 \mathrm{~kg}$ de leche/lactancia con una duración de 240 días y un contenido de grasa de 6,5-7,0\%.

En el ganado vacuno se demostró que la utilización de la producción en el día de control (PDC) posee ventajas sustanciales para la valoración de caracteres de producción de leche sobre otros métodos (Uribe y Smulders, 2004). Swalve (1995) consideró que este rasgo representó una alternativa para la selección. Igualmente indicó, que su uso tuvo la ventaja de trabajar con el registro original, donde se tenían en cuenta los efectos genéticos y ambientales específicos de cada pesaje.

Hasta el presente, en las condiciones de Cuba, se utilizaron las PDC de las búfalas solamente para calcular las lactancias totales en ganado vacuno lechero aunque en sus evaluaciones genéticas no se utilizó este criterio (Fernández, 2004; y GonzálezPeña, 2006), por lo que el objetivo de este trabajo es evaluar el comportamiento de la producción de leche y porcentaje de grasa en el día del control de las búfalas Buffalypso en la provincia Granma.

\section{MATERIAL Y MÉTODOS}

Se utilizaron 2140 pesajes de leche y 1620 de grasa de 500 lactancias desde el primer mes hasta el número 7 para leche $\mathrm{y}$ hasta el 6 para la grasa, correspondientes a 150 búfalas de la raza Buffalypso, distribuido en 6 lecherías de 3 empresas de la provincia Granma, cuyos partos se efectuaron entre los años 1997 a 2005.

A partir del décimo día de parto las búfalas se incorporaron al ordeño manual, que se realizó una vez al día, en horas de la mañana con apoyo del bucerro, al que se le dejó para su alimentación, un cuarto que se rotaba durante el ordeño.

La medición individual de la producción de leche se realizó entre 28 a 32 días, en el día del control y el ordeño se realizó a fondo, de manera que la leche que se calculó a partir de ese dato se consideró como la producción total de la hembra, sin tener en consideración la que se tomó el bucerro.

Para el estudio de las PDCL y PDCG, las producciones de leche que se registraron en el control mensual, se agruparon en dependencia de los días en leche en 7 clases de 30 días cada una para el caso de la leche (PDCL1 a PDCL 7) y de 6 para grasa (PDCG1 aPDG6).

El sistema de alimentación fundamental fue a base de pastos naturales donde predominó la jiribilla (Dichamtium caricosum), con el uso del king grass (Pennisetum pupureum) como banco de biomasa y caña de azúcar (Saccharum officinarum) como forraje además de suplemento de minerales, brindado en las corraletas, a las que tuvieron acceso sólo durante la noche. El pastoreo se realizó en horario diurno de forma continua. Esta área se encontraba acuartonada con uso de cercas eléctricas para facilitar el manejo de los animales y la mejor utilización de los pastos. 
Se empleó el PROC MIXED del SAS (2007), el modelo mixto utilizado fue el siguiente:

$$
y_{i j k m}=\mu+r_{i}+\alpha_{j}+\gamma_{1}+a_{k}(r)_{i}+(r \gamma)_{i l}+e_{i j k l m}
$$

Donde:

$\mathrm{y}_{\mathrm{ijkl}}=$ valor para cada una de las observaciones de los indicadores evaluados;

$\mu=$ media de la población;

$r_{i}=$ efecto aleatorio de rebaño $(i=1,2 \ldots 6)$;

$\alpha$ = efecto fijo de grupo de contemporáneos año de parto (1997-2005)-época de parto (en época: 1 julio-octubre y fuera de época: 2 noviembrejunio) ( $j=1,2 \ldots 18)$;

$\gamma_{1}=$ efecto fijo de fecha de pesaje (i= 1, $2 \ldots 9$ );

$a_{k}(r)_{i}=$ efecto aletorio del arete de cada animal $k$ anidado dentro de cada rebaño i;

$\left(r^{*} \gamma\right)=$ interacción rebaño*fecha de pesaje;

$\mathrm{e}_{\mathrm{ijklm}}=$ error residual, normal e independientemente distribuido $\left(0, \sigma_{e}{ }^{2}\right)$.

Para el estudio de las curvas de lactancia se utilizaron las producciones de leche y $\%$ grasa en el día del control (PDCL y PDCG). Se aplicó el modelo cuadrático logarítmico para la modelación de las curvas y como software para la representación y estimación de los términos, se empleó Stagraphics Plus. Versión 2.1.

\section{RESULTADOSYDISCUSIÓN}

Los valores de producción total de leche de forma general estuvieron entre $658 \mathrm{~kg}$ en 170 días de lactancia como promedio. Estos valores se encuentran muy por debajo a lo señalado para el país por Mitat et al. (2008) que fue de $742,3 \pm 219,5 \mathrm{~kg}$ en $229,2 \pm 36,0$ días de lactancia. Otros autores también han informado producciones de leche muy superiores a las alcanzadas aquí tales como Aspilcueta-Borquis et al. (2006) que se refirieron a tres rebaños de búfalas Murrah y sus mestizas en Brasil, donde las producciones ajustadas a 270 días fueron de $598,0 \pm 563,9 \mathrm{~kg}$. La mayor producción antes señalada pudo deberse al genotipo y mayor intensidad de selección sobre los animales menos productivos.
Las bajas producciones de leche en este estudio, son debidas en primer lugar a que a este rebaño no se le ha practicado ningún tipo de selección (positiva y negativa), otro factor fue el crecimiento continuo del número de rebaños lecheros sin que maduraran las condiciones mínimas para su explotación y la utilización de sementales no probados durante 20 años, cuya selección se efectuó de manera empírica, además de la baja y mala calidad de los alimentos suministrados en todas las categorías.

El valor estimado para la producción de grasa promedio se encuentra dentro del rango informado para Cuba es de 6,8-7,0\% (Hernández y Espinosa, 2005), así como lo indicado por otros autores en otros países: $8,16 \%$ (Verruma y Salgado, 1994); 6,8\% (Tonhati et al., 2000) y 6,11-8,15\% (Faria et al., 2002) en Brasil y al registrado por Sharma et al., 1980 (7,40\%) en India.

La media general de las PDCL fue $3,38 \pm 0,07 \mathrm{~kg}$ y variaron de acuerdo con la fase de lactación, fueron altas al inicio y disminuyeron hacia el final con valores de $4,02 \mathrm{~kg}$ a 2,36 kg (tabla I). La mayor producción se alcanzó en la PDCL2 (4,06 kg), a

Tabla I. Número de observaciones (n), medias observadas $(\bar{x})$, error estándar $(E E)$, coeficientes de variación (CV) y repetibilidad ( $\rho)$ de la producción de leche en el día de control de hembras Buffalypso en la provincia Granma. (Number of observations (n), means observed $(\bar{x})$, standard error (EE), coefficients of variation $(\mathrm{CV})$ and repeatability $(\rho)$ of milk production at control day in Buffalypso cows in Granma province).

\begin{tabular}{lccccc}
\hline Clases & $\mathrm{n}$ & $\overline{\mathrm{x}}(\mathrm{kg})$ & $\pm \mathrm{EE}$ & $\mathrm{CV}(\%)$ & $\rho$ \\
\hline PDCL1 & 369 & 4,02 & 0,07 & 34,94 & 0,21 \\
PDCL2 & 367 & 4,06 & 0,08 & 35,58 & 0,23 \\
PDCL3 & 366 & 3,80 & 0,08 & 37,46 & 0,16 \\
PDCL4 & 332 & 3,49 & 0,07 & 38,41 & 0,08 \\
PDCL5 & 291 & 3,13 & 0,07 & 39,94 & 0,14 \\
PDCL6 & 238 & 2,78 & 0,07 & 37,34 & 0,11 \\
PDCL7 & 155 & 2,36 & 0,08 & 41,67 & 0,21 \\
\hline
\end{tabular}




\section{MÉNDEZYFRAGA}

partir de la cual comenzó a declinar hasta el séptimo control. Resultados similares fueron informados por Fraga et al. (2003) y Mitat et al. (2008) para Cuba y para Brasil por Tonhati (2004), mientras que la media

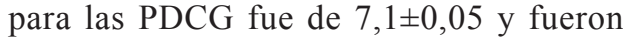
aumentando a medida que aumentaron los días de producción de leche con valores de $6,79 \%$ a $7,27 \%$ desde el primer pesaje hasta el sexto respectivamente.

Los menores coeficientes de variación se observaron al inicio de la lactancia (PDCL1 y PDCL2), como consecuencia de una menor variación en la producción de leche en ese período, posiblemente por un mejor manejo preparto y al estado corporal de las búfalas que al parir generalmente presentaron buenas reservas corporales. Al final de la lactancia aumentaron hasta $41,67 \%$ como consecuencia de una reducción muy marcada en el tamaño de la muestra y a las diferencias individuales entre los animales con relación a la persistencia de la lactancia. Resultados similares encontraron Tonhati (2004) y Mitat et al. (2008).

Las estimaciones de repetibilidad para cada PDCL fueron similares a las reportadas por Mitat et al. (2008) donde las mayores correspondieron a las PDCL9, PDCL2 y PDCL3 $(0,29 ; 0,23$ y 0,22 respectivamente) y las menores a las PDCL8 $(0,05)$ y PDCL6 $(0,13)$. Mientras que Tonhati et al. $(2000)$ en Brasil reportaron valores de 0,41 para la PLT240 (producción de leche total a 240 días de lactancia). Los valores de repetibilidad para los diferentes PDCG, variaron de 0,12 a 0,33 . El valor más elevado fue el primer control $(0,33)$ y el menor el cuarto control $(0,14)$. La repetibilidad para el porcentaje de grasa se encuentra por debajo a lo informado por Uribe y Smulders (2004) que informaron un valor de 0,43 para bovinos overos colorados.

La tendencia fue que los primeros tres controles presentaron las mayores repetibilidades. Las PDCL6 y PDCL7 fueron muy variables posiblemente como consecuencia a las características de la muestra. Mien- tras que para el porcentaje de grasa (tabla II), los mayores valores se obtuvieron en las PDCG5 y PDCG6 7,28\% y 7,27\% respectivamente.

Un aspecto importante que pudo haber influido en las variaciones es la cantidad de lactancias de cada búfala que se involucrarían en la estimación. Cuando se utilizó la primera lactancia podrían ocurrir pequeños errores en la estimación de las futuras producciones, ya que en su próximo parto la búfala tendría la influencia de nuevos factores ambientales.

Las figuras $\mathbf{1}$ y $\mathbf{2}$ muestran la curva de lactancia según el modelo cuadrático logarítmico.

El clásico pico (figura 1), se reemplaza por un pequeño ascenso alrededor de los 60 días con una producción de leche promedio para la provincia entre 4,02 y 2,36 kg/día. Posteriormente se manifestó una disminución muy ligera a lo largo de la lactancia. Similares resultados fueron encontrados por Martínez (2006) al realizar estudios en estos mismos rebaños pero sólo por dos años. Por otra parte, Fraga et al. (2003) encontraron el pico de producción a los 41 días con una producción promedio de leche de $4,87 \mathrm{~kg} /$

Tabla II. Número de observaciones (n), medias observadas $(\bar{x})$, error estándar $(E E)$ y coeficientes de variación (CV) y repetibilidad ( $\rho$ ) del \% de grasa en el día de control de hembras Buffalypso en la provincia Granma. (Number of observations (n), means observed $(\bar{x})$, standard error (EE), coefficients of variation $(\mathrm{CV})$ and repeatability $(\rho)$ of percentage of fat at control day in Buffalypso cows control females in Granma province).

\begin{tabular}{cccccc}
\hline Clases & $\mathrm{n}$ & $\overline{\mathrm{x}}(\%)$ & $\pm \mathrm{EE}$ & $\mathrm{CV}(\%)$ & $\rho$ \\
\hline PDCG1 & 289 & 6,79 & 0,05 & 12,99 & 0,33 \\
PDCG2 & 288 & 6,92 & 0,05 & 11,72 & 0,12 \\
PDCG3 & 283 & 7,09 & 0,04 & 10,59 & 0,12 \\
PDCG4 & 248 & 7,23 & 0,04 & 9,08 & 0,14 \\
PDCG5 & 217 & 7,28 & 0,04 & 8,56 & - \\
PDCG6 & 176 & 7,27 & 0,05 & 9,27 & - \\
\hline
\end{tabular}



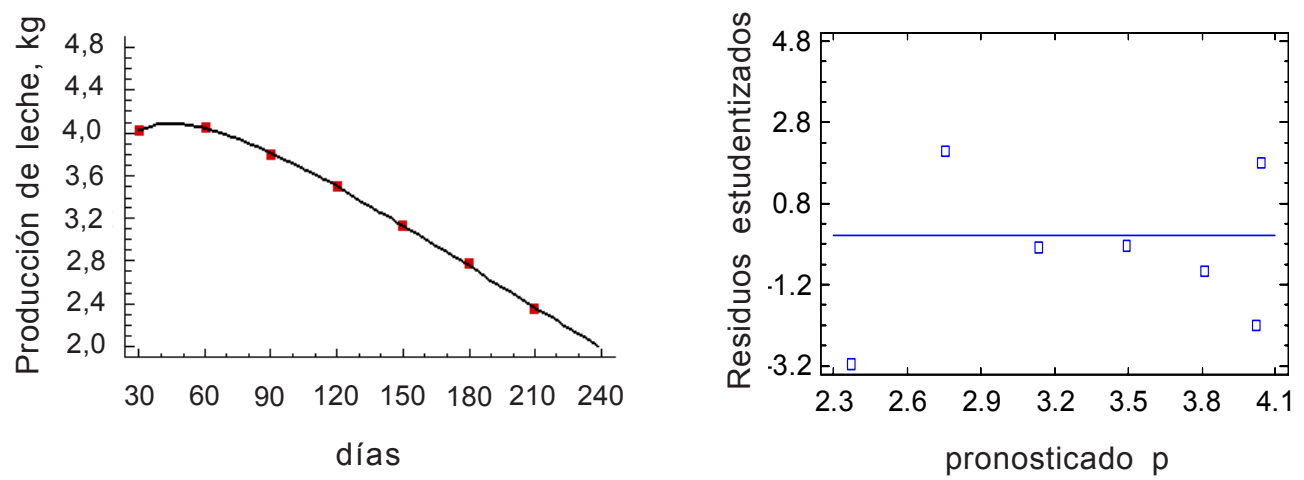

Figura 1. Curva de lactancia y análisis de los residuos para las PDCL de búfalas en la provincia Granma. (Lactation curve and residual analysis for PDCL in buffalo cows in Granma province).

día y Sedan et al. (2001), en un estudio a búfalas en el estado de Zulia, Venezuela, observaron que el pico de producción se produjo a los 51 días con una producción promedio de leche de $4,75 \mathrm{~kg} /$ día. En el caso del \% de grasa (figura 2) se inicia con un ascenso a medida que aumenta los días de lactancia, resultados similares a lo reportado por Capdevila et al. (2001) donde informan que al ir aumentando los meses de lactancia aumenta consigo el porciento de grasa.

En el caso del \% de grasa (figura 2) se inicia con un ascenso a medida que aumentan los días de lactancia, resultados simila-

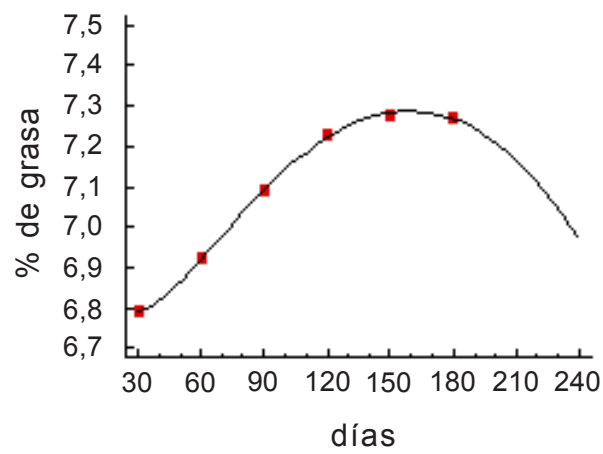

res a lo reportado por Capdevila et al.(2001) donde informan que al ir aumentando los meses de lactancia aumenta consigo el porciento de grasa.

Los ajustes de las curvas (tabla III) aportan coeficientes de determinación $\left(\mathrm{R}^{2}\right) \mathrm{y}$ coeficientes de determinación ajustado $\left(\mathrm{R}^{2} \mathrm{~A}\right)$ altos $(99,95$ y $99,96 \%),(99,90$ y $99,96 \%)$ para PDCL y PDCG respectivamente. Estos resultados son superiores a lo informado por Méndez (2007) que al utilizar varios modelos matemáticos (modelo lineal hiperbólico, Wood 1967, Alí y Schaeffer 1987, cuadrática logarítmica y modelo logístico)

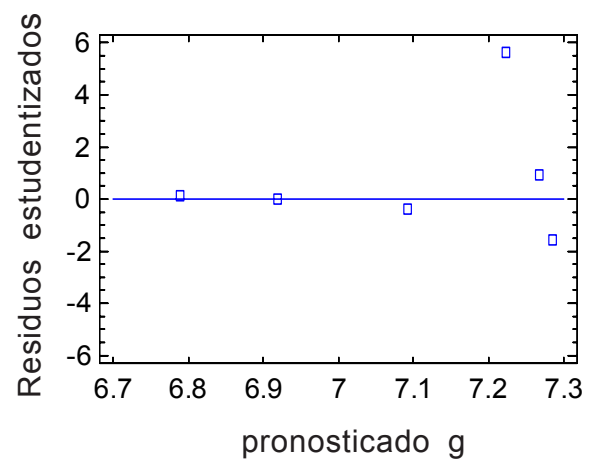

Figura 2. Curva de \% de grasa y análisis de los residuos para las PDCG de búfalas en la provincia Granma. (Fat percent curve and residual analysis of PDCG in buffalo cows in Granma province). 


\section{MÉNDEZYFRAGA}

Tabla III. Estimadores de los parámetros de las curvas de lactancia de acuerdo al modelo cuadrático logarítmico. (Parameter estimators of lactation curves according to the quadratic logarithmic model).

\begin{tabular}{lcc}
\hline Indicadores & PDCL & PDCG \\
\hline Significación del modelo & $* * *$ & $* * *$ \\
$\mathrm{R}^{2}$ & 99,95 & 99,96 \\
$\mathrm{R}^{2} \mathrm{~A}$ & 99,90 & 99,89 \\
Error estándar de la estimación & 0,02 & 0,01 \\
Media absoluta del error & 0,01 & 0,002 \\
Prueba de Durbin-Watson & 2,9 & 3,5 \\
\hline
\end{tabular}

$R^{2}$ : Coeficiente de determinación; $R^{2} A$ : Coeficiente de determinación ajustado.

el modelo cuadrático logarítmico fue el que mejor caracterizó la curva de lactancia, además estos altos coeficientes pudieron deberse al ajuste a que fueron sometidas las PDCL para los efectos considerados en el modelo de efectos mixtos descrito anteriormente. La misma tabla muestra que la media absoluta del error y el error estándar de estimación fueron inferiores tanto para leche como para la grasa. La prueba de DurbinWatson reflejó que no existen serios pro- blemas de autocorrelación de los errores. La tabla IV presenta un resumen de los parámetros estimados, EE y la significación de éstos, además de las ecuaciones resultantes para las PDCL Y PDCG.

En Cuba, estudios similares fueron realizados en un rebaño experimental por Fraga et al. (2003) y Mitat et al. (2008) donde obtuvieron que de los modelos utilizados, el cuadrático logarítmico fue el que más se ajustó para la caracterización de la curva de lactancia en esas condiciones, al igual a lo reportado por Muñoz-Berrocal et al. (2005) en el estado de Sao Paulo, Brasil, donde obtuvieron que este modelo (cuadrático logarítmico) fue el que mejor se ajustó $\left(\mathrm{R}^{2}=\right.$ $86,29)$. Otras investigaciones se han realizado en especies bovinas como es el caso del genotipo Siboney de Cuba, donde Fernández (2004), encontró que el mejor modelo que caracterizó $\left(\mathrm{R}^{2}=93,23\right)$ la curva de lactancia fue el de Ali y Schaeffer (1987) en la EPG Camilo Cienfuegos en la provincia de Pinar del Río.

\section{CONCLUSIONES}

La producción de leche en el día de control de las búfalas que se estudiaron fue

Tabla IV. Resultado del ajuste del modelo cuadrático logarítmico para PDCL y PDCG. (Adjustment results of quadratic logarithmic model for PDCL and PDCG).

\begin{tabular}{|c|c|c|c|c|}
\hline & \multicolumn{4}{|c|}{ Parámetros } \\
\hline & $a \pm E S$ & $b \pm E S$ & $c \pm E S$ & $d \pm E S$ \\
\hline PDCL & $1,50 \pm 0,40$ & $-0,022 \pm 0,003$ & $0,00001 \pm 0,00001$ & $0,93 \pm 0,14$ \\
\hline \multirow[t]{2}{*}{ PDCG } & $7,91 \pm 0,17$ & $0,020 \pm 0,002$ & $-0,000055 \pm 0,000004$ & $-0,50 \pm 0,06$ \\
\hline & \multicolumn{4}{|c|}{ Significación de los parámetros } \\
\hline PDCL & $* * *$ & $* * *$ & n.s. & *** \\
\hline \multirow[t]{2}{*}{ PDCG } & $* * *$ & *** & $* * *$ & $* \star *$ \\
\hline & \multicolumn{4}{|c|}{ Ecuaciones resultantes } \\
\hline PDCL & \multicolumn{4}{|c|}{$y=1,49776-0,0218845 * t+0,000010917 * t^{\wedge} 2+0,932876 * \log (t)$} \\
\hline PDCG & \multicolumn{4}{|c|}{$y=7,90541+0,0208431{ }^{*} t-0,000055795{ }^{*} t^{\wedge} 2-0,497064{ }^{*} \log (t)$} \\
\hline
\end{tabular}

Donde: $y=$ producción diaria de leche, $\mathrm{kg} ; \mathrm{t}=$ día de lactancia.

Archivos de zootecnia vol. 61, núm. 233, p. 16. 


\section{PRODUCCIÓN DE LECHE Y GRASA EN EL DÍA DE CONTROL DE BÚFALAS DE RÍO}

baja y el \% de grasa se mantuvo dentro del rango para esta especie. Las repetibilidades determinadas de forma general se encuentran dentro del rango de medio a alta. La modelación con los valores PDCL y PDCG ajustados por el modelo lineal tuvieron coeficientes de ajuste altos con el modelo

\section{BIBLIOGRAFÍA}

Ali, T.E. and Schaeffer, L.R. 1987. Accounting for covariances among test day milk yields in dairy cows. Can J Anim Sci, 67: 637.

Aspilcueta-Borquis, R.R., Tonhati, H., Seno, L.O., Mendoza-Sánchez, G., Sesana, R. y Albuquerque, L.G. 2006. Estimación de parámetros genéticos para producción de leche y sus constituyentes (grasa y proteína) en búfalas. Memorias $3^{\text {er }}$ Simposio de Búfalos de las Américas. Medellín. Colombia. p. 246.

Capdevila, J., Zaldivar, V. y Martínez, I. 2001. Caracterización físico-química de la leche de búfala preveniente de mezclas en Cuba. Efecto del mes y la época del año. Primer Encuentro Nacional de Criadores de Búfalos. Villa Clara.

Faria, M.H., Tohat, I.H., Ceron Muñoz, M., Duarte, J.M.C. e Vasconcellos, B.F. 2002 .Características físico-químicas do leite de búfalas ao longo da lactação. Rev Inst Laticinios "Candido Tostes", 57: 3-7.

Fernández, L. 2004. Modelos estadísticos-matemáticos en el análisis de la curva de lactancia y factores que la afectan en el genotipo Siboney de Cuba. Tesis (Doctor en Ciencias Veterinarias). Universidad Agraria. La Habana.

Fraga, L.M. 2008. Estudo do desempenho produtivo e reprodutivo de bubalinos explorados no Brasil e Cuba. Relatorio posdoctoral CAPES. FMVZ. UNESP. Botucatú-SP. Brasil.

Fraga, L.M., Gutiérrez, M., Fernández, L., Fundora, O. y González, M.E. 2003. Estudio preliminar de las curvas de lactancia en búfalas mestizas de Murrah. Rev Cubana Cienc Agric, 37: 151.

García, S. y Planas, T. 2003. Manual de crianza del búfalo. ACPA. pp. 1-9.

González-Peña, D. 2006. Evaluación genética del ganado Siboney de Cuba empleando la producción en el día de control bajo un modelo de regresión aleatoria. Tesis (Doctor en Ciencias Veterinarias). Centro de Investigación para el cuadrático logarítmico. Por lo que se recomienda realizar la selección de búfalas para producción lechera a partir de los registros repetidos del día de control por los valores más altos de repetibilidad obtenidos y estudiar los factores no genéticos que pudieran estar afectando la producción lechera.

Mejoramiento Animal. Ministerio de la Agricultura. Cuba.

Hernández, R. y Espinosa, E. 2005. Ordeño de las búfalas: Manejo, rutina y consideraciones prácticas. Nota técnica. ACPA, 1: 16-17.

Martínez, A. 2006. Caracterización del sistema de producción de lecherías bufalinas en la provincia Granma. Tesis Master en Producción Animal. ICA. La Habana. Cuba.

Méndez, M. 2007. Caracterización productiva y reproductiva del Búfalo (Bubalus bubalis) en algunos hatos en la provincia Granma. Cuba. Tesis Master en Producción Animal. ICA. La Habana. Cuba.

Mitat, A., Menéndez, A., González-Peña, D. y Ramos, F. 2008. Producción en el día de control en hembras Buffalypso y mestizas con carabao. Descripción. Rev Cienc Tecnol Gan, 2: 27-32. Muñoz-Berrocal, M.F., Tonhati, H., Cerón-Muñoz, M., Duarte, J.M.C. e Chabariberi, R.L. 2005. Uso de modelos lineares e não lineares para o estudo da curva de lactação em Búfalos Murrah e seus mestiços em sistema de criação semiextensivo no Estado de São Paulo. Arch Latinoam Prod Anim, 13: 19.

SAS. 2007. User's guide statistics. SAS Institute Inc. Cary, NC. USA.

Sedan, J.M., Díaz, Y. y Montiel, N. 2001. Efecto de factores no genéticos sobre la producción de leche en búfalas. Trabajo de recopilación de información. El búfalo: el bovino del presente y del futuro. Universidad de Zulia. Venezuela.

Sharma, U.P., Rao, S.K. and Zariwala, I.T. 1980. Composition of milk of different breeds of buffaloes. Ind J Dairy Sci, 33: 7-12.

Swalve, H.H. 1995. Genetic relationship between dairy lactation persistency and yield. J Anim Breed Genet, 112: 303-311.

Tonhati, H. 2004. Criterios de selección para búfalos lecheros. Memorias $2^{\circ}$ Simposio de Búfa- 


\section{MÉNDEZYFRAGA}

os de las Américas. Belén. Brasil.

Tonhati, H., Muñoz-Cerón, M., Oliveira, J., Duarte, J., Furtado, T. e Tseimazides, S. 2000. Parâmetros genéticos para a produção de leite, gordura e proteína em bubalinos. Rev Bras Zootecn, 29 2051-2056.

Uribe, H.A. y Smulders, J.P. 2004. Estimación de parámetros y tendencias fenotípicas, ambien- tales y genéticas para características de producción de leche en bovinos overos colorados. Arch Med Vet, 36: 137-146.

Verruma, M. e Salgado, M.J. 1994. Análise química do leite de búfala en comparação ao leite de vaca. Sci Agric Piracicaba, 51: 131-137.

Wood, P.D.O. 1967. Algebraic model of the lactation curve in cattle. Nature, 216: 164.

Archivos de zootecnia vol. 61, núm. 233, p. 18. 